Artículo científico

Volumen 30(3):767-782. Septiembre-diciembre, 2019

e-ISSN 2215-3608, doi:10.15517/am.v30i3.36103

http://www.revistas.ucr.ac.cr/index.php/agromeso

\title{
Emisión de gases de efecto invernadero en el suelo bajo el uso de abonos verdes $^{1}$
}

\section{Emission of greenhouse gases in the soil under the green manure effect}

\author{
Breno Augusto Sosa-Rodrigues ${ }^{2}$, Yuly Samanta García-Vivas ${ }^{2}$
}

1 Recepción: 6 de febrero, 2019. Aceptación: 8 de abril, 2019. Este trabajo formó parte de la tesis de doctorado realizada por el primer autor, Universidad Nacional de Colombia sede Palmira, Colombia.

2 Universidad Nacional Autónoma de Honduras (UNAH), Centro Universitario Regional del Litoral Atlántico (CURLA), detrás del Aeropuerto Internacional Goloson, La Ceiba, Atlántida, Honduras. breno.sosa@unah.edu.hn, yuly.garcia@unah.edu.hn (autor para correspondencia; https://orcid.org/0000-0001-7506-797X).

\section{Resumen}

Introducción. Las prácticas de manejo del suelo en los agroecosistemas, como el uso de abonos verdes (AV), afectan positivamente las emisiones de los gases de efecto invernadero (GEI). Objetivo. El objetivo de la investigación fue evaluar la emisión de $\mathrm{CO}_{2}, \mathrm{CH}_{4}$ y $\mathrm{N}_{2} \mathrm{O}$, de un suelo cultivado con maíz y soya, bajo el uso de abonos verdes, barbecho (B) y fertilización nitrogenada. Materiales y métodos. Entre julio de 2011 y junio de 2012, en un Typic Haplustert, ubicado en el Valle del Cauca, Colombia, se sembraron maíz y soya en forma intercalada y los monocultivos de maíz y soya, los dos últimos manejados de forma convencional. Se estableció como AV la asociación entre Mucuna pruriens var. utilis y maíz, noventa días después de la siembra, el total de los residuos se incorporaron como AV o se dispusieron sobre la superficie del suelo como acolchado orgánico (AO). Se utilizaron once tratamientos bajo el diseño de bloques completos al azar, con arreglo factorial $3^{2}+2$, con tres repeticiones. Las parcelas principales las constituyó AV, AO y B. Cada una se dividió en subparcelas que correspondieron a la fertilización por compost, fertilizante de síntesis química industrial y ninguna adición. En la etapa de floración y llenado de grano de los cultivos se analizaron variables como el $\mathrm{N}_{2} \mathrm{O}, \mathrm{CH}_{4}, \mathrm{CO}_{2}$ y porosidad total llena de agua. Resultados. El flujo $\mathrm{CO}_{2}$ fue mayor en los cultivos intercalados adicionados con AV/AO en comparación con los monocultivos. En las parcelas principales el uso de fertilizantes de síntesis química industrial estimuló significativamente los flujos de $\mathrm{N}_{2} \mathrm{O}$ y $\mathrm{CH}_{4}$. Conclusión. El uso de materiales orgánicos de alta calidad como AV/AO no aseguraron suelos sumideros de GEI a corto plazo, ya que las condiciones del suelo y la fertilización nitrogenada influyeron de forma significativa en las emisiones.

Palabras clave: Zea mays, Glycine max, óxido nitroso, metano, dióxido de carbono.

\begin{abstract}
Introduction. Soil management practices in agroecosystems, such as the use of green manures (GM), positively affect greenhouse gas (GHG) emissions. Objective. The objective of the research was to evaluate $\mathrm{CO}_{2}, \mathrm{CH}_{4}$, and $\mathrm{N}_{2} \mathrm{O}$ emission of a soil cultivated with corn and soybean under the use of green fertilizers, fallow (B), and nitrogen fertilization. Materials and methods. Between July 2011 and June 2012, in a Typic Haplustert located in Valle del Cauca, Colombia, corn and soybeans were planted in a intervleaved manner, and the monocultures of corn and
\end{abstract}


soybeans were managed in a conventional manner. The association between Mucuna pruriens var. utilis and maize was established as GM, ninety days after sowing, the total residues were incorporated as GM or disposed on the soil surface as organic mulch $(\mathrm{AO})$. Eleven treatments were used under the design of randomized complete blocks, with $3^{2}+2$ factorial arrangement, with three repetitions. The main plots were GM, AO and B. Each was divided into subplots corresponding to compost fertilization, industrial chemical synthesis fertilizer and no additions. In the flowering and grain filling stage of the crops, variables such as $\mathrm{N}_{2} \mathrm{O}, \mathrm{CH}_{4}, \mathrm{CO}_{2}$ and total water-filled porosity were analyzed. Results. $\mathrm{CO}_{2}$ flow was higher in the intercrops added with $\mathrm{GM} / \mathrm{AO}$ compared to monocultures. In the main plots, the use of industrial chemical synthesis fertilizers significantly stimulated $\mathrm{N}_{2} \mathrm{O}$ and $\mathrm{CH}_{4}$ fluxes. Conclusion. The use of high quality organic materials such as GM/AO did not ensure GHG sink soils in the short-term, since soil conditions and nitrogen fertilization significantly influenced emissions.

Keywords: Zea mays, Glycine max, nitrous oxide, methane, carbon dioxide.

\section{Introducción}

Los gases de efecto invernadero (GEI) son capaces de absorber la radiación infrarroja emitida por el suelo, lo que mantiene la temperatura media del planeta en aproximadamente $15{ }^{\circ} \mathrm{C}$ (Baird, 2002). El incremento de la concentración de los GEI en la atmósfera es causada por actividades humanas concernientes a la producción industrial y de alimentos (IPCC, 2008).

La agricultura se ha asociado con el incremento de las concentraciones de los tres principales GEI en la atmósfera, dióxido de carbono $\left(\mathrm{CO}_{2}\right)$, metano $\left(\mathrm{CH}_{4}\right)$ y óxido nitroso $\left(\mathrm{N}_{2} \mathrm{O}\right)$, los cuales, contribuyen al calentamiento global. A nivel mundial, se estima que la agricultura contribuye con $20 \%$ de las emisiones antropogénicas de GEI, aproximadamente $22 \%$ de las emisiones totales de $\mathrm{CO}_{2}, 55 \%$ de $\mathrm{CH}_{4}$ y $80 \%$ de $\mathrm{N}_{2} \mathrm{O}$ (Houghton et al., 2001; IPCC, 2008). Las causales de las emisiones de $\mathrm{CO}_{2}$ son: el cambio en el uso del suelo, deforestación y quema de biomasa. Para las emisiones de $\mathrm{N}_{2} \mathrm{O}$ son: el uso de plantas leguminosas y fertilización mineral nitrogenada. Las emisiones de $\mathrm{CH}_{4}$ suelen ocurrir en suelos con poca aireación, como en los sistemas inundados de arroz y explotación ganadera (Kessavalou et al., 1998; Costa, 2005).

En Colombia, se estima que el 38,1\% de las emisiones de $\mathrm{CO}_{2}$ equivalentes (eq), 65,92\% de $\mathrm{CH}_{4}$ y 95,56\% de $\mathrm{N}_{2} \mathrm{O}$ en $\mathrm{CO}_{2}$ eq, son originadas por el sector agropecuario (Ministerio de Ambiente, Vivienda y Desarrollo Territorial, 2009). Estas emisiones cobran mayor importancia cuando se miden por el potencial de calentamiento global (PCG), ya que el $\mathrm{CH}_{4}$ (23) y $\mathrm{N}_{2} \mathrm{O}$ (296) poseen más poder calorífico que el $\mathrm{CO}_{2}$ en un horizonte de cien años (IPCC, 2008).

Las prácticas de manejo del suelo también afectan las emisiones de los GEI. Los sistemas de preparación del suelo en los cultivos afectan el balance entre la absorción de carbono (C) fotosintetizado y las emisiones de $\mathrm{CO}_{2}$ del suelo para la atmósfera, que lleva a caracterizar al suelo como banco (absorción>emisión) o fuente (emisión>absorción) de este gas. En ese sentido, los sistemas en monocultivos tienden acumular C en forma de materia orgánica (MO), siendo mayores las tasas de acumulación cuando los sistemas son en asocio o rotación de cultivos con altos aportes de residuos vegetales (Amado et al., 2000; Diekow et al., 2005).

El uso de leguminosas en sistemas de rotación de cultivos aumentan las emisiones de $\mathrm{N}_{2} \mathrm{O}$ en comparación a sistemas exclusivamente con gramíneas (Baggs et al., 2003), a su vez, la tasas son potencialmente mayores cuando se usan fertilizantes minerales nitrogenados. De esta forma, el manejo de mezclas entre especies leguminosas y gramíneas, puede ser una alternativa de mitigación al proveer $\mathrm{N}$ al suelo desde fuentes biológicas en comparación con los métodos convencionales, así se podrían mejorar las relaciones de carbono:nitrógeno (C:N) de los residuos, reduciendo la velocidad de mineralización y consecuentes pérdidas al ambiente. 
El uso de especies que se incorporan al suelo como los abonos verdes (AV) o que se disponen en la superficie como acolchado orgánico ( $\mathrm{AO}$ ) en los agroecosistemas, son una alternativa viable al manejo de $\mathrm{C}$ y $\mathrm{N}$ en sistemas convencionales de barbecho (B), ya que aportan considerables cantidades de MO, nutrientes y agua en sus residuos (Baijukya et al., 2004; Gallego, 2012), sin embargo, las emisiones de GEI también están sujetas a características intrínsecas del suelo y condiciones ambientales, lo que dificulta el manejo integrado del fenómeno intensificado. El objetivo de la investigación fue evaluar la emisión de $\mathrm{CO}_{2}, \mathrm{CH}_{4}$ y $\mathrm{N}_{2} \mathrm{O}$ de un suelo cultivado con maíz y soya, bajo el uso y manejo de abonos verdes, barbecho y fertilización nitrogenada.

\section{Materiales y métodos}

\section{Caracterización del área experimental}

El estudio se realizó de julio del 2011 a junio del 2012, en un Haplustert típico, francoso fino, isohipertérmico, con pendiente de $1 \%$ (Acosta et al., 1997), el cual estuvo en barbecho durante ocho años. El Campo Experimental de la Universidad Nacional de Colombia (UNAC), sede Palmira (CEUNP), corregimiento El Carmelo, municipio de Candelaria, Departamento del Valle del Cauca, Colombia, está georeferenciado bajo las coordenadas $3^{\circ} 2^{\prime}$ ' 34" N y $76^{\circ} 25^{\prime} 53^{\prime \prime} \mathrm{O}$. Las características agroclimáticas que la zona presenta son una altitud de $980 \mathrm{msnm}$, temperatura promedio de $24{ }^{\circ} \mathrm{C}$, humedad relativa de $69 \%$ y precipitación pluvial media anual de $1406 \mathrm{~mm}$. La caracterización química y física del suelo, antes de instalar el ensayo, se realizó en los laboratorios de la UNAL, Palmira y el CIAT.

Los resultados de las propiedades químicas (Cuadro 1) se interpretaron por escalas manejadas a nivel nacional (Castro, 2004). El suelo presentó un $\mathrm{pH}$ neutro, concentración media de $\mathrm{CO}$ y $\mathrm{N}$ inorgánico, y alto nivel de fósforo. Entre las bases, se encontraron niveles medios de $\mathrm{Na}^{+}$y $\mathrm{K}^{+}$y altos de $\mathrm{Mg}^{2+}$ y $\mathrm{Ca}^{2+}$, además de la capacidad de intercambio catiónico (CIC). Para los elementos menores S, B y Mn los niveles fueron altos, a excepción de Fe, $\mathrm{Cu}$ y $\mathrm{Zn}$ que fueron bajos. Se ha reportado que a $\mathrm{pH}$ neutro se encuentra la mayor disponibilidad de bases (Piraneque, 2008), mientras que en condiciones ácidas la asimilación del P disminuye por combinaciones insolubles con Fe y los microelementos, a excepción del Mo, son más asimilables (Zapata, 2004; Tisdale et al., 2005).

Cuadro 1. Caracterización química del suelo a una profundidad de $0-20 \mathrm{~cm}$, utilizado para evaluar el efecto de los abonos verdes sobre la emisión de los gases de efecto invernadero (GEI). Valle del Cauca, Colombia. 2013.

Table 1. Chemical characterization of soil at a depth of $0-20 \mathrm{~cm}$, used to evaluate the effect of green fertilizers on greenhouse gas (GHG) emission. Valle del Cauca, Colombia. 2013.

\begin{tabular}{|c|c|c|c|c|c|c|c|c|c|c|c|c|c|c|c|c|}
\hline $\mathrm{CO}$ & $\mathrm{pH}_{\mathrm{H} 2 \mathrm{O}}$ & $\mathrm{N}$ total & $\mathrm{NH}_{4}^{+}$ & $\mathrm{NO}_{3}^{-}$ & P (Bray II) & $\mathbf{S}$ & B & $\mathbf{F e}$ & Mn & $\mathrm{Cu}$ & $\mathbf{Z n}$ & $\mathbf{K}$ & Ca & Mg & $\mathrm{Na}$ & CIC \\
\hline $\mathrm{g} \mathrm{kg}^{-1}$ & & ----- & $-\cdots$ & $-\cdots$ & $---m g ~ k$ & ------"- & ---- & $\cdots$ & ------" & & & \multicolumn{5}{|c|}{--------cmol kg $^{-1}$} \\
\hline 26,6 & 6,84 & $1,174,5$ & 4,42 & 13,84 & 57,45 & 28,03 & 1,45 & 8,83 & 61,75 & 0,67 & 1,49 & 0,44 & 10,9 & 5,63 & 0,10 & 21,10 \\
\hline
\end{tabular}

El análisis de las propiedades físicas (Cuadro 2) mostraron que el suelo posee una textura franco arcillosa, la cual permite una adecuada laborabilidad cuando la humedad gravimétrica no supera un $50 \%$. La densidad aparente fue alta $\left(1,72 \mathrm{~g} \mathrm{~cm}^{-3}\right) \mathrm{y}$, por ende, muy baja porosidad total $(35,34 \%)$, lo que puede perturbar no solo el crecimiento de raíces (según especie), drenaje y aireación en el suelo (Jaramillo, 2002), sino que cuando supera $60 \%$ del espacio poroso con agua, se generan condiciones de reducción y se favorece la emisión de GEI (Escobar, 2011). 
Cuadro 2. Caracterización física del suelo a una profundidad de 0-20 cm, utilizado para evaluar el efecto de los abonos verdes sobre la emisión de gases de efecto invernadero. Valle del Cauca, Colombia. 2013.

Table 2. Physical characterization of soil at a depth of 0-20 cm, used to evaluate the effect of green fertilizers on greenhouse gases emission. Valle del Cauca, Colombia. 2013.

\begin{tabular}{cc}
\hline Parámetro & Valor medio \\
\hline Densidad aparente $\left(\mathrm{g} \mathrm{cm}^{-3}\right)$ & 1,72 \\
Densidad real $\left(\mathrm{g} \mathrm{cm}^{-3}\right)$ & 2,66 \\
Porosidad total $(\%)$ & 35,34 \\
Humedad gravimétrica (\%) & 19,11 \\
Humedad volumétrica (\%) & 32,86 \\
Porosidad total llena agua (\%) & 92,9 \\
Arenas (\%) & 43,1 \\
Arcillas (\%) & 27,4 \\
Limos (\%) & 29,5 \\
Clase textural & Franco arcillosa \\
\hline
\end{tabular}

\section{Tratamientos y diseño experimental}

Para cumplir con los objetivos propuestos, se evaluaron once tratamientos (Cuadro 3), bajo un diseño de bloques completos al azar con arreglo factorial $3^{2}$ (tratamientos 1 al 9) +2 (tratamientos 10 y 11) con tres

Cuadro 3. Descripción de tratamientos utilizados para determinar el efecto de los abonos verdes sobre la emisión de gases de efecto invernadero del suelo. Valle del Cauca, Colombia. 2013.

Table 3. Description of treatments used to determine the effect of green fertilizers of soil greenhouse gases emission. Valle del Cauca, Colombia. 2013.

\begin{tabular}{llcc}
\hline Parcela principal & & Subparcela & Tratamiento* \\
\hline $\begin{array}{l}\text { 1. Abono verde (Mucuna pruriens + Zea } \\
\text { mays L.) }\end{array}$ & Cultivo intercalado de maíz y soya, tratado con abono orgánico & AV-FO \\
& Cultivo intercalado de maíz y soya, tratado con fertilizante de síntesis & AV-FQ \\
& Cultivo intercalado de maíz y soya, sin fertilización adicional & AV-SF \\
\hline 2. Acolchado orgánico (Mucuna pruriens & Cultivo intercalado de maíz y soya, tratado con abono orgánico & AO-FO \\
+ Zea mays L.) & Cultivo intercalado de maíz y soya, tratado con fertilizante de síntesis & AO-FQ & AO-SF \\
\hline 3. Barbecho & Cultivo intercalado de maíz y soya, sin fertilización adicional & B-FO \\
& Cultivo intercalado de maíz y soya, tratado con abono orgánico & B-FQ & B-SF \\
\hline Cultivo intercalado de maíz y soya, tratado con fertilizante de síntesis & B-FQ \\
& Cultivo intercalado de maíz y soya, sin fertilización adicional & B-FQ \\
\hline
\end{tabular}

La estructura factorial $3^{2}$ corresponde del tratamiento 1 al 9, sin embargo, se adicionó el tratamiento 10 y 11 (+2), convirtiéndose en un factorial aumentado $\left(3^{2}+2\right) /$ Factorial structure $3^{2}$ corresponds to treatments 1 to 9 , however treatment 10 and $11(+2)$ were added, becoming an increased factorial $\left(3^{2}+2\right)$.

* AV: abono verde; AO: acolchado orgánico; B: barbecho; FO: fertilización orgánica; FQ: fertilización de síntesis química industrial; SF: sin adición de fertilizantes / AV: green fertilizer; AO: organic mulch; B: fallow; FO: organic fertilization; FQ: fertilization of industrial chemical synthesis; SF: no fertilizer additions. 
repeticiones. Las cuatro parcelas principales las constituyó el componente vegetal [abono verde (AV) o barbecho (B)] previo al establecimiento de los cultivos, más dos con B, todos seguidos por sistemas de cultivos asociados (maíz + soya) y de monocultivos (maíz o soya). Las subparcelas correspondieron a tres diferentes programas de fertilización (compost, fertilizante de síntesis química industrial y ninguna adición).

\section{Manejo agronómico de las parcelas}

Las unidades fueron parcelas en campo con una dimensión de $20 \mathrm{~m}^{2}$ (6 m x 3,34 m) para los tratamientos del 1 al 9 , y de $30 \mathrm{~m}^{2}(6 \mathrm{~m}$ x $5 \mathrm{~m})$ para los tratamientos 10 y 11. Posteriormente, se instaló un pluviómetro y termómetro (Figura 1), con el fin de estimar la lámina de riego que cayó sobre el suelo (precipitación pluvial + riego) y la temperatura ambiental de la zona durante el desarrollo del experimento.

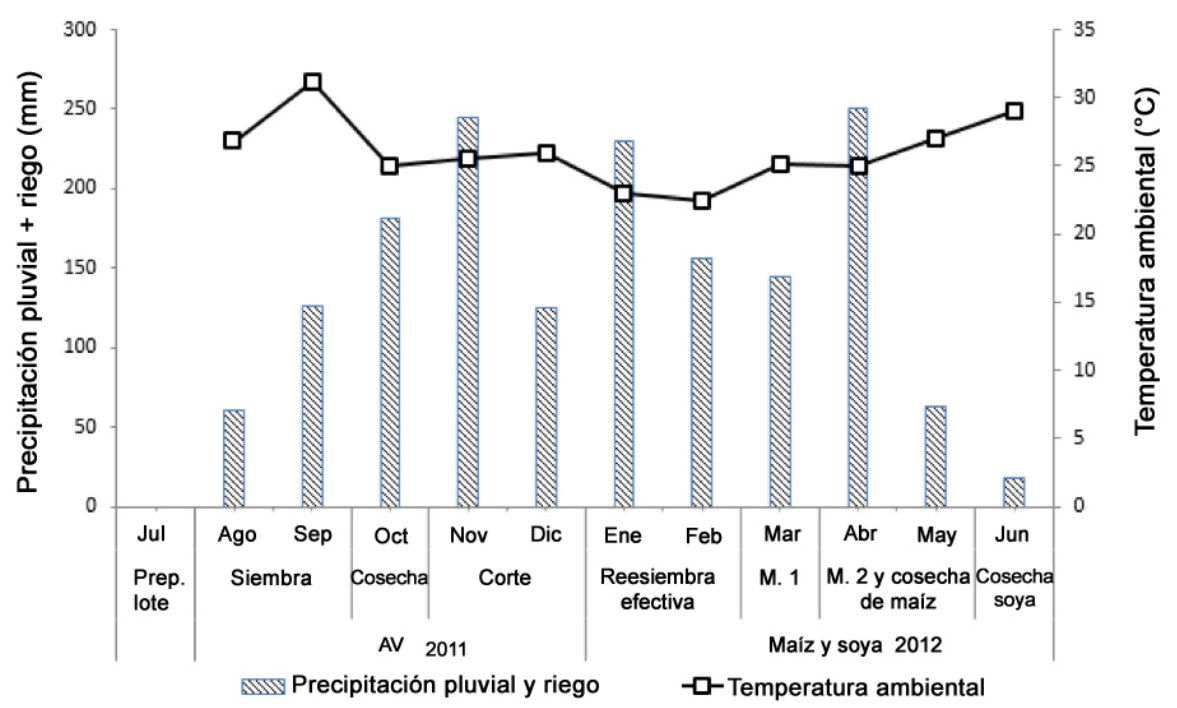

Figura 1. Distribución mensual de la temperatura ambiental y lámina de agua en el suelo (precipitación pluvial + riego) durante el experimento para determinación del efecto de los abonos verdes sobre la emisión de gases de efecto invernadero del suelo. Valle del Cauca, Colombia. 2013.

Figure 1. Monthly distribution of the environmental temperature and water sheet in the soil (rainfall + irrigation) during the experiment to determine the effect of the green fertilizers on the soil GHG emission. Valle del Cauca, Colombia. 2013.

Después del trazado de las unidades experimentales, se sembró como AV la leguminosa M. pruriens L. var. utilis (CIAT No. 9349) y Z. mays (variedad ICA 305) en las unidades experimentales correspondientes a los tratamientos 1 a 6 , a una dosis de $110 \mathrm{~kg}$ semilla $\mathrm{ha}^{-1}$. Se dejaron crecer por un período aproximado de tres a cuatro meses, hasta la etapa reproductiva del llenado de grano lechoso (R3) de la gramínea (etapa de choclo). Luego de la cosecha del choclo, la biomasa (adición o incorporación) de los AV se cortó, troceó y dejó descomponer en los primeros cinco centímetros del suelo por treinta días.

El establecimiento del cultivo de maíz (variedad ICA 305) se realizó con una densidad de siembra de 40000 plantas ha-1 ${ }^{-1}$ mientras la siembra de soya (variedad ICA P34) se realizó con 200000 plantas ha-1 , con una relación de un surco de maíz por dos de soya en cada unidad experimental. 
Las fertilizaciones con fuentes orgánicas y sintéticas se realizaron en dos momentos, a los 15 y 45 días de la resiembra efectiva de los cultivos. La fertilización sintética se realizó aplicando el fertilizante triple quince (1515-15) a una dosis de $50 \mathrm{~kg} \mathrm{~N}, \mathrm{P}_{\text {y K ha }}{ }^{-1}$ (335 g y $501 \mathrm{~g}$ por aplicación en parcelas de intercultivo y monocultivo, respectivamente), de acuerdo con los requerimientos de los cultivos (Moreno-Berrocal et al., 2008). Para los tratamientos con adición de abono orgánico tipo compost, la dosis se calculó para que aportara la misma cantidad de $\mathrm{N}$ que el fertilizante sintético, en ese sentido, este consistió en gallinaza madura aplicada en dosis de 3,4 tha ${ }^{-1}$ (3,4 kg por aplicación), lo que equivale a $50 \mathrm{~kg} \mathrm{~N} \mathrm{ha}^{-1}$, y el resto de tratamientos se manejó sin la aplicación de fertilizantes o enmiendas.

\section{Variables evaluadas}

El flujo de los gases de efecto invernadero $\left(\mathrm{CO}_{2}, \mathrm{CH}_{4}, \mathrm{~N}_{2} \mathrm{O}\right)$ y su poder calorífico, se analizó directamente y se relacionó con variables como compactación y circulación del agua en el suelo, las cuales hacen parte de la fórmula de la porosidad total llena de agua (PPA) (Cuadro 4).

Cuadro 4. Métodos de análisis de las variables utilizadas para evaluar el efecto de los abonos verdes sobre la emisión de gases de efecto invernadero del suelo. Valle del Cauca, Colombia. 2013.

Table 4. Analysis methods of the variables used to evaluate the effect of green fertilizers of soil greenhouse gases emissions. Valle del Cauca, Colombia. 2013.

\begin{tabular}{lcc}
\hline Determinaciones & Métodos & Referencia \\
\hline $\mathrm{CO}_{2}\left(\mathrm{~kg} \mathrm{~m}^{-2}\right)$ & Cámara cerrada estática & Ferreira (2008) \\
$\mathrm{CH}_{4} \mathrm{y} \mathrm{N}_{2} \mathrm{O}\left(\mathrm{mg} \mathrm{m}^{-2}\right)$ & Gravimetría & \\
\hline Humedad gravimétrica (\%) & Fórmula matemática & \\
Humedad volumétrica (\%) & Cilindro biselado & Jaramillo (2002) \\
Densidad aparente $\left(\mathrm{g} \mathrm{cm}^{-3}\right)$ & Pícnómetro & \\
Densidad real $\left(\mathrm{g} \mathrm{cm}^{-3}\right)$ & Fórmula matemática & \\
Porosidad total $(\%)$ & Fórmula matemática & Escobar (2011) \\
\hline Porosidad total llena de agua (PPA \%) & Fórmula matemática & Gomes (2006) \\
\hline Potencial Calentamiento Global & & \\
\hline PGG $\left(\mathrm{kg} \mathrm{CE}-\mathrm{CO}_{2}\right)$ &
\end{tabular}

Para la toma de los gases, realizada en la etapa de floración y llenado de grano de los cultivos, se procedió a ejecutar la técnica de cámara cerrada estática, protocolo estandarizado por el Laboratorio de Servicios Ambientales del Centro Internacional de Agricultura Tropical (CIAT), utilizado por Ferreira (2008) y Pastrana et al. (2011). Se inició con la instalación de una cámara de PVC sobre el anillo previamente instalado en la parcela de cada tratamiento, las cámaras se cerraron herméticamente con bandas de caucho. La parte superior de estas poseían una tapa de goma o séptum de $1 \mathrm{~cm}$ de diámetro, por la cual se tomaron las muestras de aire a las 9 de la mañana, utilizando una jeringa de plástico con válvula adaptada.

Por cada cámara se tomaron muestras de $20 \mathrm{ml}$ de aire en los tiempos $0,10,20$ y $30 \mathrm{~min}$ a partir de la instalación de la cámara, luego se eliminaron $5 \mathrm{ml}$ y los restantes $15 \mathrm{ml}$ se transfierieron a frascos al vacío. Es necesario conocer la temperatura del aire dentro de la cámara al momento del muestreo para poder calcular 
adecuadamente los flujos de gases, en ese sentido, la primera cámara en cada tratamiento estuvo equipada con un termómetro para registrar los valores para cada uno de los tiempos.

Las muestras se enviaron al laboratorio del CIAT, donde se estimaron las concentraciones de los GEI, con el uso de un cromatógrafo de gases Shimadzu GC-14A, equipado con detectores de inonización de llama (FID) en el análisis de $\mathrm{CH}_{4}$ y detector de captura de electrones (ECD) para el $\mathrm{N}_{2} \mathrm{O}$. Las condiciones del cromatógrafo fueron: precolumna Porapak Q (80-100 mesh) con $1 \mathrm{~m}$ de longitud y $2 \mathrm{~mm}$ de diámetro interno; columna Porapak Q (80$100 \mathrm{mesh}$ ) con $2 \mathrm{~m}$ de longitud y $2 \mathrm{~mm}$ de diámetro interno; fase móvil nitrógeno de alta pureza; flujo de $22 \mathrm{ml}$ $\min ^{-1}$; temperatura del horno, inyector y detector: 70,80 y $320^{\circ} \mathrm{C}$, respectivamente; estándar Scotty balanceado en nitrógeno con una precisión de aproximadamente $5 \%$.

Para el cálculo del $\mathrm{CO}_{2}$ se utilizó un analizador Modelo S151 con tecnología infrarroja, calibrado antes de iniciar las mediciones con estándares Scotty balanceados en nitrógeno. Se usó sílica gel para retener humedad y una trampa de $\mathrm{NaOH}$ para asegurar que solo el $\mathrm{CO}_{2}$ proveniente de la muestra ingresare al equipo y evitar sobreestimación en la cuantificación.

Los muestreos en el suelo para las variables relacionadas a la emisión de GEI, se realizaron en forma paralela a la toma de los gases; en cada etapa (floración y llenado de grano de cultivos) se recolectaron o midieron muestras para evaluar directamente la humedad y densidad del suelo, otras se calcularon de forma indirecta (fórmula). A partir de los valores de densidad y humedad gravimétrica se calculó el porcentaje de PPA mediante la ecuación (Escobar, 2011):

$\operatorname{PPA}(\%)=\frac{\left(\mathrm{H}_{\mathrm{g}} \times \mathrm{Da}\right)}{P_{T}}$

Donde:

$\mathrm{H}_{\mathrm{g}}$ : humedad gravimétrica $(\%)$.

$\mathrm{D}_{\mathrm{a}}$ : densidad aparente del suelo $\left(\mathrm{g} \mathrm{cm}^{-3}\right)$.

$\mathrm{P}_{\mathrm{T}}$ : porosidad total del suelo (\%).

Para la comparación de sistemas de manejo por el PCG que genera, es común expresar la emisión de GEI en $\mathrm{kg}$ de $\mathrm{C}$ equivalente de $\mathrm{CO}_{2}\left(\mathrm{~kg} \mathrm{CE}-\mathrm{CO}_{2}\right)$, el PCG de $\mathrm{CH}_{4}$ y $\mathrm{N}_{2} \mathrm{O}$ expresada en $\mathrm{CE}-\mathrm{CO}_{2}$ se calcularon a partir de las ecuaciones 2 y 3 , respectivamente:

$\mathrm{CH}_{4}\left(\mathrm{~kg} \mathrm{CE}-\mathrm{CO}_{2} \mathrm{ha}^{-1}\right)=\mathrm{CH}_{4}\left(\mathrm{~kg} \mathrm{CH}_{4} \mathrm{ha}^{-1}\right) \times 23 \times 12 / 44$

$\mathrm{N}_{2} \mathrm{O}\left(\mathrm{kg} \mathrm{CE}-\mathrm{CO}_{2} \mathrm{ha}^{-1}\right)=\mathrm{N}_{2} \mathrm{O}\left(\mathrm{kg} \mathrm{N}_{2} \mathrm{O}\right.$ ha $\left.^{-1}\right) \times 296 \times 12 / 44$

Donde:

12/44= razón molar entre $\mathrm{C}$ y la molecular de $\mathrm{CO}_{2}$

23 y 296= PCG de $\mathrm{CH}_{4}$ y $\mathrm{N}_{2} \mathrm{O}$, respectivamente, en un tiempo de cien años (Gomes, 2006; Escobar, 2011).

\section{Análisis de la información}

La información obtenida se sometió a un análisis de varianza ( $\mathrm{p}<0,05)$, prueba de medias de Duncan $(\mathrm{p}<0,05)$ y correlación de Pearson, con el uso del software SAS versión 9.1.3 (SAS Institute Inc., 2002). 


\section{Resultados}

\section{Cambios en las emisiones de $\mathrm{CO}_{2}, \mathrm{CH}_{4} \mathrm{y} \mathrm{N}_{2} \mathrm{O}$}

Se encontraron diferencias significativas $(\mathrm{p}<0,05)$ entre tratamientos para el flujo de $\mathrm{CO}_{2}$ en las etapas productivas de los cultivos (Figura 2). Los valores a nivel de parcelas principales (AV, AO y B) y subparcelas (FO, FQ y SF) fueron estadísticamente similares, con excepción de B-FQ en el monocultivo de soya, donde se registró el menor valor.

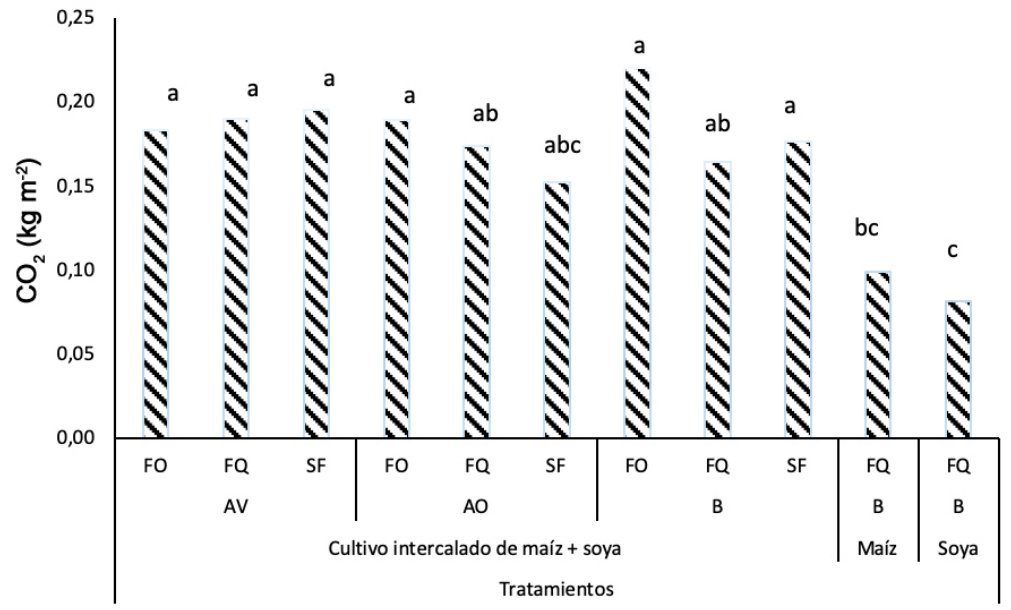

Figura 2. Flujo acumulado de $\mathrm{CO}_{2}$ en los tratamientos al evaluar el efecto de los abonos verdes sobre la emisión de gases de efecto invernadero del suelo. Valle del Cauca, Colombia. 2013.

Promedios con las mismas letras no son significativamente diferentes según Duncan $(\mathrm{p}<0,05)$. AV: abono verde; AO: acolchado orgánico; B: barbecho; FO: fertilización orgánica; FQ: fertilización de síntesis química industrial; SF: sin adición de fertilizantes.

Figure 2. Cumulative $\mathrm{CO}_{2}$ flow in the treatments when evaluating the effect of green fertilizers on the emission of greenhouse gases of the soil. Valle del Cauca, Colombia. 2013.

Averages with the same letters are not significantly different according to Duncan ( $\mathrm{p}<0.05)$. GM: green manure; AO: organic mulch; B: fallow; FO: organic fertilization; FQ: fertilization of industrial chemical synthesis; SF: no fertilizer additions.

En el flujo de $\mathrm{CH}_{4}$ se encontraron diferencias significativas $(\mathrm{p}<0,05)$ entre tratamientos (Figura 3 ). En todas las parcelas los resultados variaron entre valores negativos y positivos $\left(-23,3\right.$ a 40,8 $\left.\mathrm{mg} \mathrm{m}^{-2}\right)$, lo cual denotó cambios en la capacidad del suelo como sumidero y emisor del $\mathrm{CH}_{4}$, respectivamente. Aún con esta dinámica se vio reflejada alguna tendencia, las parcelas de $\mathrm{AV}$ y AO con fertilización orgánica actuaron como sumideros, mientras que la fertilización de síntesis industrial (FQ) promovió la emisión en AV y B.

Para la variable $\mathrm{N}_{2} \mathrm{O}$ se encontraron diferencias significativas $(\mathrm{p}<0,05)$ entre tratamientos (Figura 4). En las parcelas principales AV, AO y B bajo la combinación con FQ se estimuló los mayores flujos de $\mathrm{N}_{2} \mathrm{O}$ en comparación a FO.

Se registró mayor poder calorífico del $\mathrm{CO}_{2}$ emitido del suelo en todos los tratamientos con respecto a los otros gases, y el de $\mathrm{N}_{2} \mathrm{O}$ fue mayor a los registrados del $\mathrm{CH}_{4}$ en la mayoría de las parcelas (Figura 5).

El $\mathrm{CO}_{2}$ contribuyó con el 99,7 \% de los GEI totales del agroecosistema (Figura 6), seguido del óxido nitroso y metano, aunque el que genera mayor potencial de calentamiento global es el $\mathrm{N}_{2} \mathrm{O}$, es común encontrar esta secuencia en varios suelos agrícolas. 


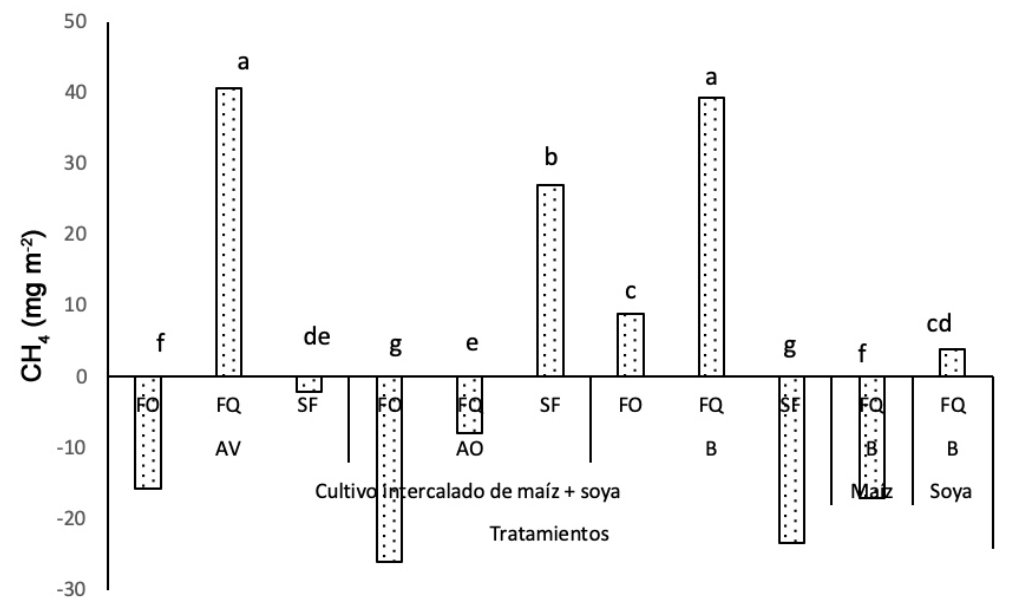

Figura 3. Flujo acumulado de $\mathrm{CH}_{4}$ en los tratamientos al evaluar el efecto de los abonos verdes sobre la emisión de gases de efecto invernadero del suelo. Valle del Cauca, Colombia. 2013.

Promedios con las mismas letras no son significativamente diferentes según Duncan ( $<<0,05)$. AV: abono verde; AO: acolchado orgánico; B: barbecho; FO: fertilización orgánica; FQ: fertilización de síntesis química industrial; SF: sin adición de fertilizantes.

Figure 3. Cumulative flow of $\mathrm{CH}_{4}$ in the treatments when evaluating the effect of green fertilizers on the emission of greenhouse gases of the soil. Valle del Cauca, Colombia. 2013.

Averages with the same letters are not significantly different according to Duncan ( $\mathrm{p}<0.05)$. GM: green manure; AO: organic mulch; B: fallow; FO: organic fertilization; FQ: fertilization of industrial chemical synthesis; SF: no fertilizer additions.

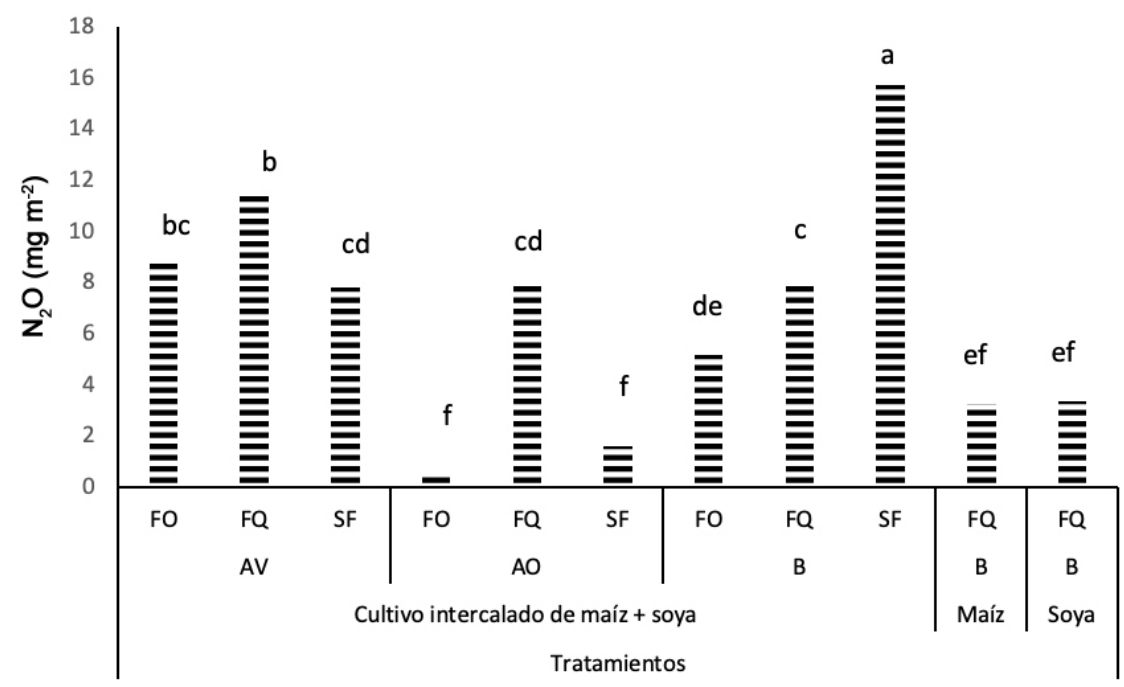

Figura 4. Flujo acumulado de $\mathrm{N}_{2} \mathrm{O}$ en los tratamientos al evaluar el efecto de los abonos verdes sobre la emisión de gases de efecto invernadero del suelo. Valle del Cauca, Colombia. 2013.

Promedios con las mismas letras no son significativamente diferentes según Duncan $(\mathrm{p}<0,05)$. AV: abono verde; AO: acolchado orgánico; B: barbecho; FO: fertilización orgánica; FQ: fertilización de síntesis química industrial; SF: sin adición de fertilizantes.

Figure 4. Accumulated $\mathrm{N}_{2} \mathrm{O}$ flow in the treatments when evaluating the effect of green fertilizers on the emission of greenhouse gases of the soil. Valle del Cauca, Colombia. 2013.

Averages with the same letters are not significantly different according to Duncan ( $\mathrm{p}<0.05)$. GM: green manures; AO: organic mulch; B: fallow; FO: organic fertilization; FQ: fertilization of industrial chemical synthesis; SF: no fertilizer additions. 


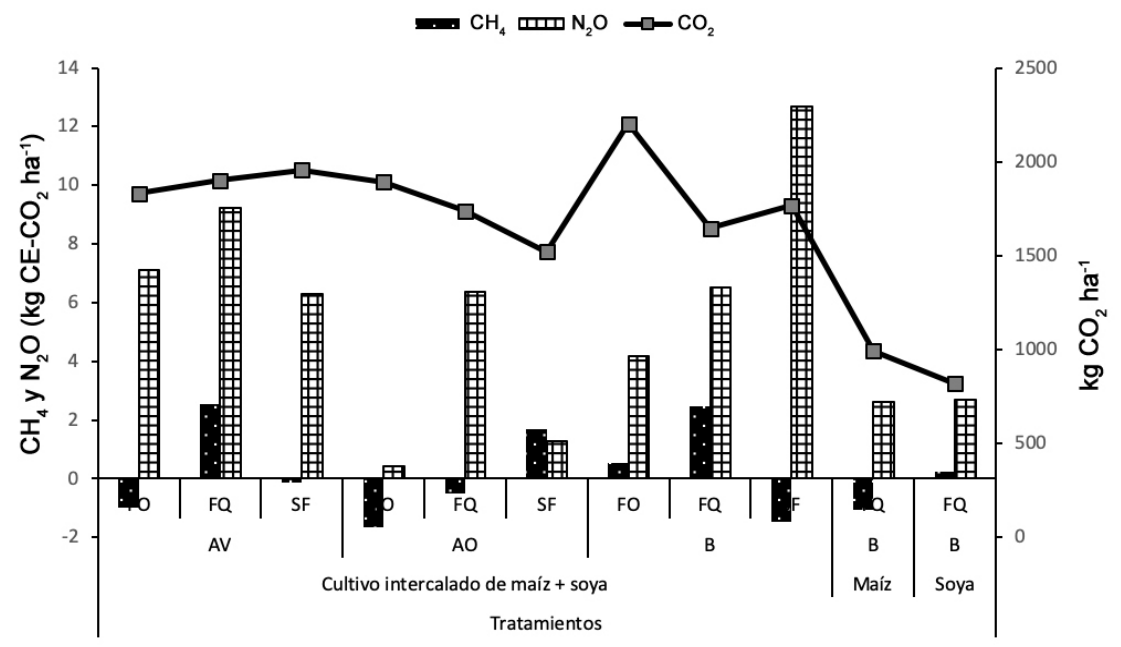

Figura 5. Potencial del calentamiento global de los gases en los tratamientos al evaluar el efecto de los abonos verdes sobre la emisión de gases de efecto invernadero del suelo. Valle del Cauca, Colombia. 2013.

AV: abono verde; AO: acolchado orgánico; B: barbecho; FO: fertilización orgánica; FQ: fertilización de síntesis química industrial; SF: sin adición de fertilizantes.

Figure 5. Global warming potential of the gases in the treatments when evaluating the effect of the green fertilizers on the greenhouse gases emission of the soil. Valle del Cauca, Colombia. 2013.

GM: green manures; AO: organic mulch; B: fallow; FO: organic fertilization; FQ: fertilization of industrial chemical synthesis; SF: no fertilizer additions.

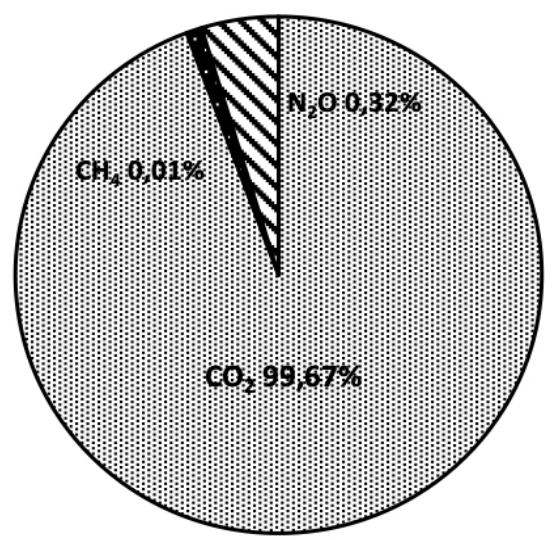

Figura 6. Aporte porcentual de cada gas emitido del suelo en el estudio, efecto de los abonos verdes sobre la emisión de gases de efecto invernadero del suelo. Valle del Cauca, Colombia. 2013.

Figure 6. Percentage contribution of each gas emitted from the soil in the study; effect of green fertilizers on the emission of greenhouse gases of the soil. Valle del Cauca, Colombia. 2013.

\section{Porosidad total llena de agua}

No se encontraron diferencias significativas entre tratamientos $(\mathrm{p}<0,05)$ en la porosidad total llena de agua (Figura 7), debido a la variabilidad en los datos obtenidos durante este ensayo, además, el análisis de correlación de Pearson no mostró relación significativa alguna entre esta variable y cada uno de los GEI analizados en este experimento. 


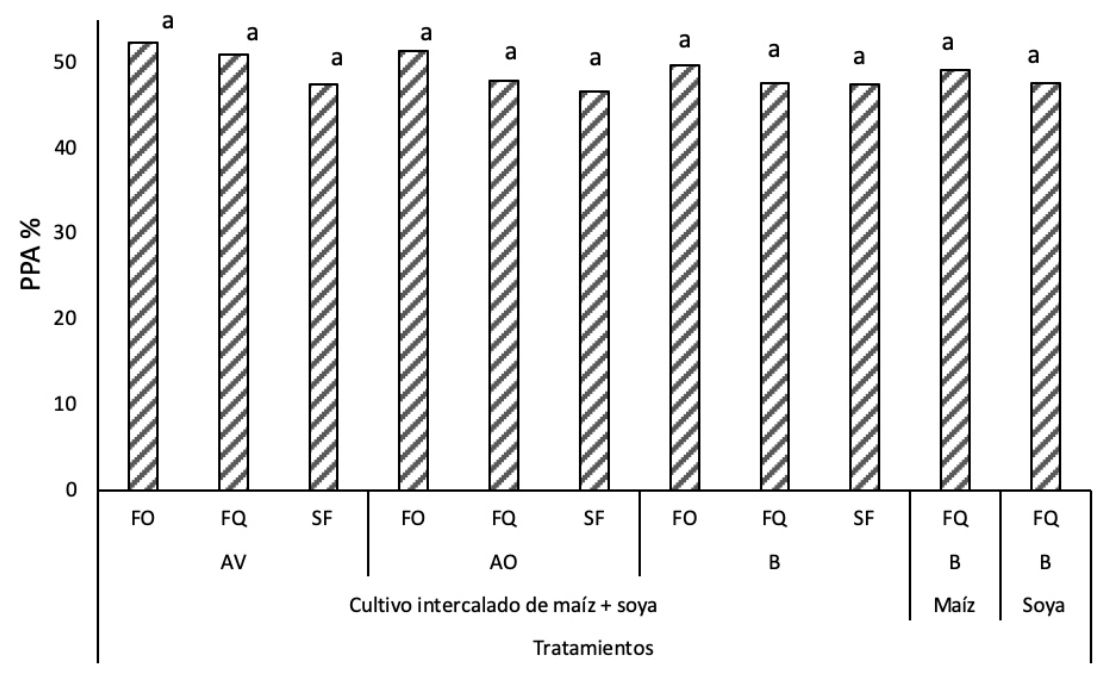

Figura 7. Porosidad total llena de agua (PPA) en los tratamientos al evaluar el efecto de los abonos verdes sobre la emisión de gases de efecto invernadero del suelo. Valle del Cauca, Colombia. 2013.

Promedios con las mismas letras no son significativamente diferentes según Duncan $(\mathrm{P}<0,05)$. AV: abono verde; AO: acolchado orgánico; B: barbecho; FO: fertilización orgánica; FQ: fertilización de síntesis química industrial; SF: sin adición de fertilizantes.

Figure 7. Total porosity full of water (PPA) in the treatments when evaluating the effect of green fertilizers on the emission of greenhouse gases of the soil. Valle del Cauca, Colombia. 2013.

Averages with the same letters are not significantly different according to Duncan ( $\mathrm{P}<0.05)$. GM: green manures; AO: organic mulch; B: fallow; FO: organic fertilization; FQ: fertilization of industrial chemical synthesis; SF: no fertilizer additions.

\section{Comparaciones específicas entre variables relacionadas al flujo de GEI}

Las comparaciones entre las fuentes de variación con respecto a algunas variables (Cuadro 5), indicaron que no existieron diferencias significativas para las variables $\mathrm{CO}_{2}$ y PPA, por el contrario, se incrementó significativamente

Cuadro 5. Contrastes ortogonales para gases de efecto invernadero y porosidad del suelo llena de agua (PPA) en el estudio sobre los efectos de los abonos verdes sobre la emisión de gases de efecto invernadero del suelo. Valle del Cauca, Colombia. 2013.

Table 5. Orthogonal contrasts for greenhouse gases and porosity of water-filled soil (PPA) in the study on the effects of green manures on the emission of greenhouse gases of the soil. Valle del Cauca, Colombia. 2013.

\begin{tabular}{|c|c|c|c|c|}
\hline \multirow[b]{2}{*}{ Fuente de variación } & \multirow{2}{*}{$\begin{array}{c}\mathrm{CO}_{2} \\
\mathrm{~kg} \mathrm{~m}^{-2}\end{array}$} & $\mathrm{CH}_{4}$ & $\mathrm{~N}_{2} \mathrm{O}$ & \multirow{2}{*}{$\begin{array}{c}\text { PPA } \\
\%\end{array}$} \\
\hline & & & & \\
\hline $\mathrm{AV}$ vrs $\mathrm{AO}$ & ns & * & $*$ & ns \\
\hline AV vrs B & ns & ns & ns & ns \\
\hline $\mathrm{AO}$ vrs $\mathrm{B}$ & ns & $*$ & $*$ & ns \\
\hline FO-SF vrs FQ en AV & ns & $*$ & $*$ & ns \\
\hline FO-SF vrs FQ en AO & ns & $*$ & $*$ & ns \\
\hline FO-SF vrs FQ en B & ns & $*$ & $*$ & ns \\
\hline
\end{tabular}

AV: abono verdes; AO: acolchado orgánico; B: barbecho; FO: fertilización orgánica; FQ: fertilización de síntesis química industrial; SF: sin adición de fertilizantes. *: existe diferencia significativa, ns: no significativo / AV: green manure; AO: organic mulch; B: fallow; FO: organic fertilization; FQ: fertilization of industrial chemical synthesis; SF: no fertilizers additions. *: there is a significant difference, ns: not significant. 
la producción de $\mathrm{CH}_{4}$ y $\mathrm{N}_{2} \mathrm{O}$ cuando se agregaron materiales orgánicos ( $\mathrm{AV}$ y B) al suelo, en comparación a $\mathrm{AO}$, principalmente bajo la condición $\mathrm{FQ}$.

\section{Discusión}

En los cultivos intercalados los tratamientos generaron efectos similares en el flujo de $\mathrm{CO}_{2}$, en este sistema se encontraron mayores flujos que en los monocultivos (Franzluebbers, 2005; Adiku et al., 2010). Los mismos autores indicaron que es posible que la mayor cobertura en la superficie del suelo, a la cual estuvo sujeta al sembrar cultivos intercalados, permitió mayor respiración autotrófica y heterotrófica, lo que originó la liberación del $\mathrm{CO}_{2}$, tanto de los sistemas radiculares de las plantas como de la actividad de organismos en el suelo al descomponer la compleja $\mathrm{MO}$ en compuestos más simples. Los flujos acumulados de $\mathrm{CO}_{2}$ encontrados en este ensayo $\left(0,08\right.$ a $\left.0,22 \mathrm{~kg} \mathrm{~m}^{-2}\right)$, coincidieron e incluso fueron menores que los obtenidos por Ferreira (2008) y Fernández y Bojollo (2008), los cuales oscilaron entre 0,07 y $2,2 \mathrm{~kg} \mathrm{~m}^{-2} \mathrm{año}^{-1}$.

El flujo de $\mathrm{CH}_{4}$ fue fluctuante, caracterizado por parcelas con la capacidad de ser sumidero y emisor del gas. Se

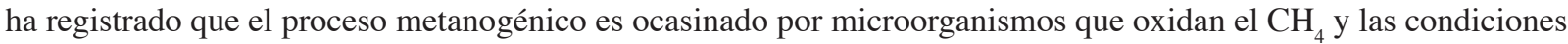
del suelo, entre ellas, la textura, humedad y porosidad (Hütsch et al., 1994; 1996). Este proceso, se suscita cuando prevalecen condiciones anaeróbicas en el suelo y las poblaciones de bacterias metanogénicas transforman el ácido acético, $\mathrm{H}_{2}$ y $\mathrm{CO}_{2}$ en $\mathrm{CH}_{4}$ (Weber et al., 1984). Caso contrario sucede cuando el medio es más aeróbico, las bacterias metanotróficas oxidan el $\mathrm{CH}_{4}$ y el suelo actúa como sumidero (Jones y Morita, 1983; Glaztel y Stahr, 2001). Kessavalou et al. (1998) demostraron que la perturbación del suelo durante el establecimiento de cultivos aumenta la oxidación del $\mathrm{CH}_{4}$, en el siguiente orden: campo nativo (33 a $\left.44 \mu \mathrm{g} \mathrm{m}^{-2} \mathrm{~h}^{-1}\right)>$ siembra directa (31 a 34 $\left.\mu \mathrm{g} \mathrm{m}^{-2} \mathrm{~h}^{-1}\right)>$ preparación convencional $\left(24,5\right.$ a $\left.33 \mu \mathrm{g} \mathrm{m}^{-2} \mathrm{~h}^{-1}\right)$.

En general, las condiciones del suelo y prácticas agrícolas también influyen en la emisión de $\mathrm{CH}_{4}$ (Ussiri et al., 2009); nótese, que no existió un patrón definido en la emisión del gas, lo cual pudo deberse tanto a las diferentes condiciones ambientales, antes mencionadas, como a la disponibilidad de $\mathrm{N}$ del suelo, la densidad aparente (varió entre 1,1 y $1,7 \mathrm{~g} \mathrm{~cm}^{-3}$ ) y la humedad volumétrica (varió entre 17 y $33 \%$ ) en las diferentes parcelas durante las etapas de evaluación; factores que han sido señalados como influyentes en la absorción o emisión de $\mathrm{CH}_{4}$ (Czóbel et al., 2010; Escobar, 2011; Omonode et al., 2011).

Entre las prácticas agronómicas, la fertilización nitrogenada regula la absorción del $\mathrm{CH}_{4}$. En algunos suelos la oxidación de $\mathrm{CH}_{4}$ disminuyó, ya que el $\mathrm{NH}_{4}^{+}$disponible actuó como inhibidor de la reacción porque compite con el $\mathrm{CH}_{4}$ por la enzima mono-oxigenasa, producida vía oxidación del $\mathrm{NH}_{4}^{+}$(Boeckx et al., 1997; Wang e Ineson, 2003; Zanatta et al., 2010). En trabajos realizados por otros autores se confirmó que esta práctica redujo de forma directa la oxidación del $\mathrm{CH}_{4}$ por la inhibición competitiva e indirectamente, a largo plazo, la acidificación del suelo modifica el pH óptimo para el establecimiento de poblaciones metanotróficas (Hütsch, 1998; Kravchenko, 2002). Sin embargo, para Glaztel y Stahr (2001), esta práctica agrícola no afectó la emisión del gas, y obtuvieron valores muy cercanos de emisión en un experimento conducido durante diez años, al comparar el efecto de la fertilización nitrogenada y sin fertilización. Cuando se compararon los tratamientos que recibieron diferentes dosis de urea, no se obtuvo efecto alguno en la oxidación de $\mathrm{CH}_{4}$ (Ruser et al., 1998).

En el presente estudio se pudo verificar la dinámica del $\mathrm{CH}_{4}$ en el suelo, expresado de forma significativa en la absorción o emisión del gas, entre los factores señalados están la disponibilidad del $\mathrm{N}$ vía fertilización, las propiedades físicas como la textura, la humedad y la porosidad que condicionan la actividad de los microorganismos

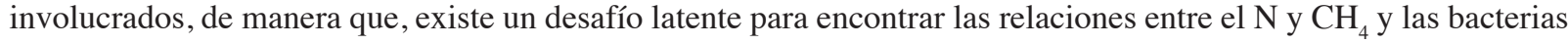
intermediarias en el proceso. 
En las parcelas con FQ existió mayor desnitrificación que las manejadas con FO, tendencia similar a la registrada para el proceso de nitrificación, especialmente en la etapa de floración de los cultivos. Varias investigaciones en sistemas de producción agrícola demostraron que el $\mathrm{N}_{2} \mathrm{O}$ emitido es respuesta a la fertilización mineral nitrogenada (Kaye et al., 2004; Omonode et al., 2011), más cuando esta sucede en periodos lluviosos (Ferreira, 2008; Pastrana et al., 2011; Escobar, 2011). Aparte de los cambios en la humedad y temperatura del suelo, el flujo de $\mathrm{N}_{2} \mathrm{O}$ también es influenciado por la disponibilidad de N, pH y la textura del suelo (Horváth et al., 2008; Czóbel et al., 2010).

En sistemas agroforestales de Honduras, el flujo de $\mathrm{N}_{2} \mathrm{O}$ mostró valores alrededor de 300 y $620 \mathrm{mg} \mathrm{m}^{-2}$ (Ferreira, 2008). En Colombia, cultivos de arroz emitieron flujos de $300 \mathrm{mg} \mathrm{N}_{2} \mathrm{O} \mathrm{m}^{2}$ año-1 (Rondón et al., 2002), mientras que, en pasturas de Brachiaria humidicola en el valle del Sinú se reportaron valores menores de $4 \mathrm{mg} \mathrm{m}^{-2} \mathrm{~h}^{-1}$ (Pastrana et al., 2011). En suelos desnudos y sembrados con soya del Valle del Cauca encontraron valores que no superaron los $420 \mathrm{mg} \mathrm{N}_{2} \mathrm{O} \mathrm{m}^{-2}$ año $^{-1}$ (Subbarao et al., 2009).

Los resultados corroboran que la metanogénesis y desnitrificación son procesos influenciados por la mineralización de la MO, fertilización mineral nitrogenada y condiciones del suelo, en especial la humedad. La disposición de la $\mathrm{MO}$ sobre el suelo (AO), generó menor emisión de $\mathrm{CH}_{4}$ y $\mathrm{N}_{2} \mathrm{O}$ en comparación a $\mathrm{AV}$ y $\mathrm{B}$. Al manejar la $\mathrm{MO}$ en forma de acolchado, se redujo la velocidad de la mineralización, por el menor contacto del sustrato con la biota del suelo en comparación a AV, paralelamente, mejoró la circulación del agua y aire en el suelo, regulando de forma más estable el suministro de los nutrimentos $\mathrm{C}$ y $\mathrm{N}$ como la variación de la temperatura y humedad (Smets y Poesen, 2009; Zribi et al., 2011).

La producción de GEI está estrechamente relacionada con la actividad de microorganismos y condiciones del suelo como la humedad, densidad y porosidad (Horváth et al., 2008; Ciarlo, 2009; Czóbel et al., 2010), de tal manera que los tratamientos influenciaron de forma similar el flujo de $\mathrm{CO}_{2}$ y PPA, y si bien existió alguna coherencia en los resultados obtenidos entre la disponibilidad de $\mathrm{N}$ mineral con el flujo de $\mathrm{N}_{2} \mathrm{O}$ (Kaye et al., 2004; Omonode et al., 2011; Pastrana et al., 2011), para el $\mathrm{CH}_{4}$ fue fluctuante como lo señalan resultados de trabajos realizados por Hellebrand et al. (2003), Dunfield (2007), Visscher et al. (2007) y Zanatta et al. (2010).

\section{Conclusiones}

El flujo de $\mathrm{CO}_{2}$ se incrementó en los cultivos intercalados adicionados con AV/AO en comparación con los monocultivos. En las parcelas principales: abonos verdes, acolchado orgánico y barbecho, el uso de fertilizantes de síntesis química industrial estimuló significativamente los flujos de $\mathrm{N}_{2} \mathrm{O}$ y $\mathrm{CH}_{4}$. Estos resultados señalan la necesidad de planificar y ejecutar sistemas de cultivos con mayor complejidad y duración que, además, de ventajas socio-económicas, y actúen como sumideros de $\mathrm{C}$ y N.

\section{Agradecimientos}

Los autores expresan su agradecimiento al Grupo de Investigación en Agroecología de la Universidad Nacional de Colombia, Sede Palmira, quien financió y aportó los recursos necesarios para esta investigación.

\section{Literatura citada}

Acosta, R.J., M. Aragón, Y. Llanos, y E. Madero. 1997. Cartografía ultradetallada de suelos y evaluación de tierras del centro experimental de la Universidad Nacional de Colombia (CEUNP) Sede Palmira. Acta Agron. 47:23-34. 
Adiku, S.G.K., N.K.Amon, J.W. Jones, T.A.Adjadeh,F.K.Kumaga, G.N. Dowuona, and E.K. Nartey. 2010. Simple formulation of the soil water effect on residue decomposition. Comm. Soil Sci. Plant Anal. 41:267-276. doi:10.1080/00103620903460781

Amado, T.J.C., J. Mielniczuk, e S.B.V. Fernandes. 2000. Legumonisas e adubação mineral como fontes de nitrogênio para o milho em sistemas de preparo de solo. Rev. Bras. Ciênc. Solo 24:179-186. doi:10.1590/S0100-06832000000100020

Baggs, E.M., M. Stevenson, A. Pihlatic, A. Regar, H. Cook, and G. Cadisch. 2003. Nitrous oxide emissions following application of residues and fertilizer under zero and conventional tillage. Plant Soil 254:361-270. doi:10.1023/A:102559312

Baijukya, F., N. de-Ridder, and K. Giller. 2004. Nitrogen release from decomposing residues of leguminous cover crops and their effect on maize yield on depleted soils of Bukoba District, Tanzania. Plant Soil 279:77-93. doi:10.1007/s11104-0052504-0

Baird, C. 2002. Química Ambiental. 2ª ed. Bookman, Porto Alegre, BRA.

Boeckx, P., O. Van-Cleemput, and I. Villaralvo. 1997. Methane oxidation in soils with different textures and land use. Nutr. Cycling Agroecosyst. 49:91-95. doi:10.1023/A:1009706324386

Castro, H. 2004. Propuesta guía de indicadores analíticos para calificar suelos estables y en proceso de degradación desde el punto de vista físico. En: CIAT, editor, Memorias I Taller Nacional sobre indicadores de calidad del suelo. CIAT, Palmira, Valle, COL. p. 37-42.

Ciarlo, E. 2009. Controles biológicos y no biológicos de las emisiones de gases nitrogenados (N2O y N2) en suelos agrícolas y ganaderos. Tesis Dr., Universidad de Buenos Aires, ARG.

Costa, F.S. 2005. Estoques de carbono orgânico e efluxos de dióxido de carbono e metano de solos em preparo convencional e plantio direto no subtrópico brasileiro. Tese Dou., Universidad Federal do Rio grande do Sul, Porto Alegre, BRA.

Czóbel, S., L. Horváth, O. Szirmai, J. Balogh, K. Pintér, Z. Nemeth, Z. Ürmös, B. Grosz, and Z. Tuba. 2010. Comparison of N2O and CH4 fluxes from Pannonian natural ecosystems. Eur. J. Soil Sci. 61:671-682. doi:10.1111/j.1365-2389.2010.01275.x

Diekow, J., J. Mileniczuk, H. Knicker, C. Bayer, D.P. Dick, and I. Kögel-Knabner. 2005. Soil C and N stocks affected by cropping systems and nitrogen fertilization in a southern Brasil Acrisol managed under no-tillage for 17 years. Soil Till. Res. 81:87-95. doi:10.1016/j.still.2004.05.003

Dunfield, P.F. 2007. The soil methane sink. In: D.S. Reay et al., editors, Greenhouse gas sinks. CAB International, Wallingford, GBR. p. 152-170.

Escobar, A.L. 2011. Mitigação das emissões de gases de efeito estufa por sistemas conservacionistas de manejo de solo. Tese Dou., Universidade Federal do Rio Grande do Sul, BRA.

Fernández, R.M.O., y R.C. Bojollo. 2008. Influencia de la climatología y el manejo del suelo en las emisiones de CO2 en un suelo arcilloso de la Vega de Carmona. Carel 6:2339-2354.

Ferreira, O. 2008. Flujos de gases de efecto invernadero, potencial de calentamiento global y evaluación de emergía del sistema agroforestal quesungual en el sur de Lempira, Honduras. Tesis MSc., Universidad Nacional de Colombia, Palmira, COL.

Franzluebbers, A.J. 2005. Soil organic carbon sequestration and agricultural greenhouse gas emissions in the southeastern USA. Soil Till. Res. 83:120-147. doi:10.1016/j.still.2005.02.012

Gallego, J.M. 2012. Efectos de dos abonos verdes sobre la mineralización del nitrógeno y la dinámica de las bacterias oxidantes del amoniaco y del nitrito en un ciclo de cultivo de maíz (Zea mays L.). Tesis MSc., Universidad Nacional de Colombia, COL.

Glatzel, S., and K. Stahr. 2001. Methane and nitrous oxide exchange in differently fertilized grassland in southern Germany. Plant Soil 231:21-35. doi:10.1023/A:1010315416866 
Gomes, J. 2006. Emissão de gases do efeito estufa e mitigação do potencial de aquecimento por sistemas conservacionistas de manejo do solo. Tese Dou., Universidade Feferal do Rio Grande do Sul, Porto Alegre, BRA.

Hellebrand, H.J., J. Kern, and V. Scholz. 2003. Long-term studies on greenhouse gas fluxes during cultivation of energy crops on sandy soils. Atmospheric Environ. 37:1635-1644. doi:10.1016/S1352-2310(03)00015-3

Horváth, L., B. Grosz, A. Machon, J. Balogh, K. Pintér, and S. Czóbel. 2008. Influence of soil type on $\mathrm{N}_{2} \mathrm{O}$ and $\mathrm{CH}_{4}$ soil fluxes in Hungarian grasslands. Community Ecol. 9(S1):75-80. doi:10.1556/ComEc.9.2008.S.11

Houghton, T., D.J. Ding, M. Griggs, P.J. Noguer, J.D. Van-der-linden, and D. Xiaosu. (eds.) 2001. Climate change 2001: The scientific basic. IPCC, GBR.

Hütsch, B.W. 1998. Tillage and land use effects on methane oxidation rates and their vertical profiles in soil. Biol. Fert. Soils 27:284-292. doi:10.1007/s003740050435

Hütsch, B.W., P. Russell, and K. Mengel. 1996. $\mathrm{CH}_{4}$ oxidation in two temperate arable soils as affected by nitrate and ammonium application. Biol. Fert. Soils 23:86-92. doi:10.1007/BF00335823

Hütsch, B.W., C.P. Webster, and D.S. Powlson. 1994. Methane oxidation in soil as affected by land use, soil pH, and N fertilization. Soil Biol. Biochem. 26:1613-1622. doi:10.1016/0038-0717(94)90313-1

Ministerio de Ambiente, Vivienda y Desarrollo Territorial. 2009. Inventario nacional de gases de efecto invernadero 2000 y 2004. Ministerio de Ambiente, Vivienda y Desarrollo Territorial, COL.

IPCC. 2008. Summary for policymakers. In: The Core Writing Team et al., editors, Climate change 2007: Synthesis report of fourth assessment report of the Intergovernmental Panel on Climate Change. IPCC, Geneva, SUI. p. 1-22.

Jaramillo, D. 2002. Introducción a la ciencia del suelo. Universidad Nacional de Colombia, COL.

Jones, R.D., and R.Y. Morita. 1983. Methane oxidation by Nitrosococcus oceanus and Nitrosomonas europea. App. Environ. Microbiol. 45:401-410.

Kaye, J.P., I.C. Burke, A.R. Mosier, and J.P. Guerschman. 2004. Methane and nitrous oxide fluxes from urban soils to the atmosphere. Ecol. Appl. 14:975-981. doi:10.1890/03-5115

Kessavalou, A., A.R. Moiser, J.W. Doran, R.A. Drijber, D.J. Lyon, and O. Heinemeyer. 1998. Fluxes of carbon dioxide, nitrous oxide and methane in Grass sod and winter wheat-fallow tillage management. J. Environ. Qual. 27:1094-1104. doi:10.2134/jeq1998.00472425002700050015x

Kravchenko, I.K. 2002. Methane oxidation in boreal peat soils treated with various nitrogen compounds. Plant Soil 242:157162. doi:10.1023/A:1019614613381

Moreno-Berrocal, A.M., L.A. Narro-León, H. Vanegas-Angarita, C.E. Molina-Gómez, J.G. Ospina-Rojas, y M. Agudelo. 2008. Respuesta del maíz a la fertilización química en la zona cafetera central de Colombia. Cenicafé 59(1):75-80.

Omonode, R.A., D.R. Smith, A. Gál, and T.J. Vyn. 2011. Soil nitrous oxide emissions in corn following three decades of tillage and rotation treatments. SSSAJ 75:152-163. doi:10.2136/sssaj2009.0147

Pastrana, I., S. Reza, M. Espinosa, E. Suárez, y E. Díaz. 2011. Efecto de la fertilización nitrogenada en la dinámica del óxido nitroso y metano en Brachiaria humidicola (Rendle) Schweickerdt. Cienc. Tecnol. Agropecu. 12:134-142. doi:10.21930/ rcta.vol12_num2_art:223

Piraneque, N.V. 2008. Factores edafológicos que determinan la presencia y diseminación del hongo Sclerotium cepivorum en el cultivo de cebolla de bulbo (Allium cepa) en Tibasosa, Boyacá. Tesis Dr., Universidad Nacional de Colombia, Palmira, COL.

Rondón, M., K. Duxbury, and R. Thomas. 2002. Effects of land use change in the Llanos of Colombia on fluxes of methane and nitrous oxide and on radiativa forcing of the atmosphere. In: CIAT, editor, Confronting global climate change. Annual report of the climate change project of CIAT. CIAT, Cali, COL. p.45-68. 
Ruser, R., H. Flessa, R. Schilling, H. Steindl, and F. Beese. 1998. Soil compaction and fertilization effects on nitrous oxide and methane fluxes in potato fields. SSSAJ 62:1587-1595. doi:10.2136/sssaj1998.03615995006200060016x

SAS Institute Inc. 2002. User`s guide Versión 9.1.3. SAS Institute Inc., Cary, NC, USA.

Smets, T., and J. Poesen. 2009. Impacts of soil tilth on the effectiveness of biological geotextiles in reducing runoff and interrill erosion. Soil Till. Res. 103:356-363. doi:10.1016/j.still.2008.11.001

Subbarao, G.V., K. Nakahara, M.P. Huertado, H. Ono, D.E. Moreta, A.F. Salcedo, A.T. Yoshihashi, T. Ishikawa, M. Ishitani, M. Ohnishi-Kameyana, M. Yoshida, M. Rondon, I.M. Rao, C.E. Lascano, W.L. Berry, and O. Ito. 2009. Evidence for biological nitrification inhibition in Brachiaria pastures. PNAS 106:17302-17307. doi:10.1073/pnas.0903694106

Tisdale, S.L., J.L. Havlin, D.B. Beaton, and L.N. Werner. 2005. Soil fertility and fertilizers. An introduction to nutrient management. $7^{\text {th }}$ ed. Prentice Hall, NJ, USA.

Ussiri, D.A.N., R. Lal, and M.K. Jarecki. 2009. Nitrous oxide and methane emissions from long-term tillage under a continuous corn cropping system in Ohio. Soil Till. Res. 104:247-255. doi:10.1016/j.still.2009.03.001

Visscher, A., P. Boeckx, and O. Cleemput. 2007. Artificial methane sinks. In: D.S. Reay et al., editors, Greenhouse gas sinks. CAB International, Wallingford, GBR. p. 184-200.

Wang, Z.P., and P. Ineson. 2003. Methane oxidation in a temperate coniferous forest soil: effects of inorganic N. Soil Biol. Biochem. 35:427-433. doi:10.1016/S0038-0717(02)00294-8

Weber, H., K.D. Kulbe, W. Chimiel, and W. Trosch. 1984. Microbial acetate conversion to methanes kinetics, yield and pathway in a two-step digestion process. Appl. Microbiol. Biotechnol. 19:224-228. doi:10.1007/BF00251840

Zanatta, J.A., C. Bayer, F.C. Vieira, J. Gomes, and M. Tomazi. 2010. Nitrous oxide and methane fluxes in south Brazilian gleysol as affected by nitrogen fertilizers. Rev. Bras. Ciênc. Solo 34:1653-1665. doi:10.1590/S0100-06832010000500018

Zapata, R. 2004. Química de la acidez del suelo. Impresión Cargraphics Impresion Digital, Cali, COL.

Zribi, W., J.M. Faci, y R. Aragüés. 2011. Efectos del acolchado sobre la humedad, temperatura, estructura y salinidad de suelos agrícolas. ATEA 107:148-162. 